\title{
Neue Ansätze zur Wirkmusteraufklärung pflanzlicher Mehrstoffgemische
}

\author{
Johanna M. Gostner Peter Gruber Kathrin Becker Martina Naschberger Florian Überall \\ Biozentrum, Sektion für Medizinische Biochemie, Medizinische Universität Innsbruck, Österreich
}

\begin{abstract}
Schlüsselwörter
Mehrstoffgemisch · Mikroarray · Expressionsanalyse . Ginkgo biloba · Padma 28 - Echinacea · Phytotherapeutika
\end{abstract}

\section{Zusammenfassung}

Obwohl die Hauptwirkungen traditioneller Präparate größtenteils seit langem bekannt sind, sind die molekularen Hintergründe der Aktivitätsmechanismen häufig nicht im Detail erforscht. Die pharmakorelevanten Aktivitäten botanischer Therapeutika resultieren oft aus der additiven oder synergistischen Wirkung einer Vielzahl von Inhaltsstoffen. Unterschiedliche Studien wurden publiziert, in denen die Effekte solcher Mehrkomponentengemische an ausgewählten Invitro-Zellmodellen mittels Genexpressionsanalyse untersucht wurden. Hier erwähnte Beispiele umfassen Transkriptionsanalysen mit Extrakten aus Ginkgo biloba und Echinacea sowie der Tibetischen Rezeptur Padma 28. Transkriptionsprofile können verwendet werden, um Moleküle und Signalwege herauszufiltern, die durch eine bestimmte Behandlung beeinflusst sind. Differenziell exprimierte Gensets können mit Informationen aus Interaktionsdatenbanken integriert werden, um Einsichten in betroffene biologische Prozesse zu erhalten. Die Methode der Transkriptionsanalyse mittels Mikroarray-Technologie ist in unterschiedlichen Bereichen der Naturstoff-Forschung anzutreffen, wobei die Einsatzgebiete von der Wirkmusterbeschreibung bis hin zum Toxizitätsprofiling reichen.

\section{Hintergrund}

Viele in traditionellen Medizinsystemen verwendete Rezepturen sind Mehrkomponentengemische. Im Gegensatz zu Monosubstanzen interagieren die Inhaltsstoffe bei komplex zusammengesetzten Rezepturen häufig additiv, nicht selten auch synergistisch, und die Wirkung beruht auf den Effekten vieler, oft

Hinweis: Padma 28 (Swissmedic No. 58436) ist in der Schweiz auch unter den Namen Padmed Circosan (Swissmedic No. 60131) und Arteria-vita (Swissmedic No. 62863) erhältlich und kassenzulässig.

\section{Keywords}

Multi-component mixture - Microarray · Expression analysis - Ginkgo biloba · Padma 28 - Echinacea · Herbal drugs

\section{Summary \\ New Approaches to Elucidate the Activities of Botanical Multi-Component Mixtures}

Although the major activities of traditional remedies have mostly been known since ancient times, their molecular mechanisms of action have usually not been investigated in much detail. The pharmaceutically relevant activities of botanical therapeutics frequently result from additive or synergistic effects of a multitude of components. Several studies have been published that analyze the effects of complex preparations on selected in vitro model cell systems by using gene expression analysis. Herein, the examples referred to include transcriptional studies with extracts from Ginkgo biloba and Echinacea as well as the Tibetan Formula Padma 28. Transcriptional profiles can be used to deduce key molecules and pathways affected upon treatment. Differentially expressed gene sets can further be integrated with information derived from interaction databases, thus giving a more comprehensive view of activated biological processes. Transcriptomics, by using microarray technology, is used as a tool in different fields of natural product research, ranging from activity monitoring to toxicity profiling.

schwacher Aktivitäten [1,2]. Daher muss die ganze Mischung als pharmakologisch aktiver Wirkstoffkomplex angesehen werden, dem eine pleiotrope Wirksignatur zugeordnet werden kann [3]. Die Effekte werden auf unterschiedliche Zielmoleküle und Zellstrukturen ausgeübt [4]. Dieses Multi-Target-Prinzip sowie die unterschiedlichen Charakterisierungen und Begrifflichkeiten von Phytotherapeutika und Krankheitsbildern im traditionellen und im modernen Kontext machen die wissenschaftliche Erforschung traditioneller Vielstoffrezepturen schwierig [5].

Vorteile erwartet man sich von pleiotropen Wirkmechanismen insbesondere bei der Behandlung komplexer, multikausaler Erkrankungen und chronischer Prozesse wie Entzündungen, die

\section{KARGER \\ Fax +497614520714 \\ Information@Karger.com}

www.karger.com
(C) 2013 S. Karger GmbH, Freiburg

$1661-4119 / 13 / 0208-0041 \$ 38.00 / 0$

Accessible online at:

www.karger.com/fok
Ao. Univ.-Prof. Mag. Dr. rer. nat. Florian Überall

Biozentrum, Sektion für Medizinische Biochemie

Medizinische Universität Innsbruck

Innrain 80-82, 6020 Innsbruck, Österreich

florian.ueberall@i-med.ac.at 
eine Vielzahl an fehlgeleiteten molekularen Signalwegen auf unterschiedlichen Ebenen involvieren.

Für eine Vielzahl von Phytochemikalien und pflanzlichen Extrakten sind immunmodulierende Aktivitäten beschrieben [6-8]. Auch antioxidative Eigenschaften werden häufig untersucht. In vielen Studien wird aber zunehmend der Einfluss auf die zelluläre Signalweiterleitung diskutiert [9].

Die alleinige Analyse der Einzelkomponenten pflanzlicher Zubereitungen liefert kein hinreichendes Funktionsbild [10]. Auch bei Vorhandensein von pharmakokinetischen und pharmakodynamischen Daten für einige in der Mischung enthaltene Einzelstoffe kann nur selten ein Rückschluss auf die Gesamtwirkung gemacht werden. Aus der Diversität an Inhaltsstoffen ergeben sich spezielle Anforderungen an die Qualitätsbeurteilung und die Erfassung von Wirksamkeit und Sicherheit [5], da toxikologische und pharmakologische Standardanalysen nur bedingt zum Einsatz gebracht werden können.

\section{Transkriptionsanalyse an In-vitro-Zellmodellen als möglicher Ansatz zur Wirkbeschreibung}

Anstelle eines reduktionistischen Forschungsansatzes werden vermehrt «Omics»-Technologien verwendet, um einen Überblick über die Vielzahl an Wirkungen von komplexen Mehrkomponentengemischen auf Transkriptions-, Metabolom- oder Proteinlevel zu erhalten $[11,12]$. Eine der meistverwendeten Methoden ist die Transkriptionsanalyse mittels Mikroarray-Technologie, die auch zur Aufklärung der Wirkung von Monosubstanz-Medikamenten herangezogen wird. Derartige Forschungsansätze eröffnen neue Möglichkeiten der Wirkbeschreibung und können so zum Verständnis von traditionell überlieferten Wirkungen beitragen oder auch mögliche neue Anwendungsfelder aufzeigen [13, 14].

Mittlerweile gibt es bereits einige publizierte Studien über Mikroarray-basierte Transkriptionsanalysen in unterschiedlichen Zellmodellen nach Behandlung mit pflanzlichen Mischungen. 3 ausgewählte Beispiele werden im Folgenden beschrieben.

\section{Ginkgo biloba-Extrakt beeinflusst Apoptose-Signalwege} in PC12-Zellen

Extrakte aus Blättern des Ginkgo-biloba-Baumes werden als Nahrungsergänzungsmittel zur Steigerung der Gedächtnisleistung vertrieben. Der standardisierte Extrakt EGb761 ${ }^{\circledR}$ wurde in klinischen Studien auf seine Wirksamkeit hinsichtlich der Verbesserung von Wahrnehmung und Erinnerung bei Patienten mit Alzheimer-Erkrankung, anderen Demenzformen, milder kognitiver Einschränkung und vaskulären Erkrankungen überprüft [15]. In unterschiedlichen Zell- und Tiermodellen wurden antientzündliche sowie neuroprotektive Effekte beschrieben.

Smith et al. [16] untersuchten die Effekte von EGb761 und ausgewählten Phytochemikalien aus Ginkgo biloba in der von Ratten stammenden PC12-Zelllinie, die aus einem Phäochromozytom des Nebennierenmarks isoliert worden war und ein Standardmodell für die Analyse neurorelevanter Mechanismen darstellt. In der Transkriptionsanalyse zeigte sich der Einfluss der EGb761-Behandlung auf Apoptose-relevante Gene. Die Interferenz des Extrakts mit der zellulären Apoptosemaschinerie könnte zumindest teilweise für die multiplen neuroprotektiven Effekte verantwortlich sein. Zusätzlich wurde der Einfluss auf die Cytochrom-c-Freisetzung, mitochondriale Funktionen und DNA-Schädigung untersucht [16]. Weiterführende Experi- mente zur endgültigen Klärung der In-vivo-Relevanz einer möglichen EGb761-vermittelten Neuroprotektion stehen noch aus.

\section{Komplexe Signalweiterleitung der pflanzlichen Tibetischen \\ Rezeptur Padma ${ }^{\circledR} 28$ in HepG2-Zellen}

Die Tibetische Rezeptur Padma 28 (Swissmedic Nr. 58436) besteht aus 20 Arzneipflanzen, natürlichem Kampfer und Kalziumsulfat [17]. Klinische Studien beschreiben eine Wirkung bei peripherer arterieller Verschlusskrankheit (PAVK) sowie bei anderen arteriosklerotischen und chronisch-entzündlichen Erkrankungen [18, 19]. In-vitro-Untersuchungen in unterschiedlichen Zellmodellen zeigten diverse biochemische Wirkmechanismen auf, unter anderem die Aktivierung von Gefäßendothelzellen und den Einfluss auf Monozytenadhäsion, Gerinnung und Thrombozytenaggregation, oxidative Modifizierung von Low-Density-Lipoprotein(LDL)-Cholesterin und den Tryptophanhaushalt sowie auf entzündliche Signalkaskaden [20].

In der Studie von Klein et al. [17] wurde die humane Hepatomzelllinie HepG2 verwendet, ein Leberzellmodell, das häufig in pharmakologischen und toxikologischen Studien eingesetzt wird. Der Einfluss von Padma 28 auf die Zellviabilität und seine antioxidative Wirkung in mit reaktiven Sauerstoffspezies (ROS) gestressten Zellen wurden bestimmt. Zudem wurde die Erhöhung der indirekten zellulären antioxidativen Kapazität durch ARE(antioxidant response element)-vermittelte Genexpression in CellSensor ${ }^{\circledR}$ ARE-bla HepG2-Reporterzellen gemessen.

Die Transkriptionsanalyse zeigte den Einfluss von Padma 28 auf die Expression von Genen, die in Phase-I- und -II-Detoxifikation involviert sind, und auf Signalwege, die für die Antwort auf oxidative Stressereignisse verantwortlich sind. Eine Netzwerkanalyse identifizierte miteinander in Wechselbeziehung stehende Netzwerke, deren zugeordnete biologische Funktionen Metabolisierungsvorgänge, molekularen Transport, interzelluläre Signalweiterleitung und Zell-Zell-Interaktionen umfassten. Einige der identifizierten Signalkaskaden sind relevant für Entwicklungsprozesse und Morphogenese oder sind in pathologische Konditionen und Entzündung involviert. Eines der Netzwerke nahm eine zentralere Position ein, da es über die meisten Querverbindungen zu weiteren Netzwerken verfügte. Den beteiligten Molekülen ist unter anderem eine Rolle in der Entwicklung und Funktion des kardiovaskulären Systems und in der Entzündungsantwort zugeordnet [17].

Die genomweite Untersuchung der transkriptionellen Veränderungen nach Padma-28-Behandlung in HepG2-Zellen konnte sowohl bekannte Mechanismen bestätigen als auch neue Signalwege identifizieren, die Grundlagen für weitere Studien liefern können.

\section{Immunmodulierende Effekte von Echinacea spp. in dendritischen Zellen}

Die Extrakte der traditionellen Heilpflanze Echinacea werden in der nordamerikanischen Volksmedizin zur Behandlung von Entzündungen des Hals- und Rachenbereichs verwendet. In-vitro- und auch In-vivo-Studien weisen auf immunmodulierende bzw. immunstimulierende Funktionen hin [21].

Wang et al. [22] analysierten den Einfluss von EchinaceaExtrakten auf humane unreife dendritische Zellen (DCs), die aus mononukleären Zellen des peripheren Blutes isoliert worden sind. DCs sind antigenpräsentierende Zellen, die eine wichtige Rolle in der angeborenen und adaptiven Immunität spielen. 


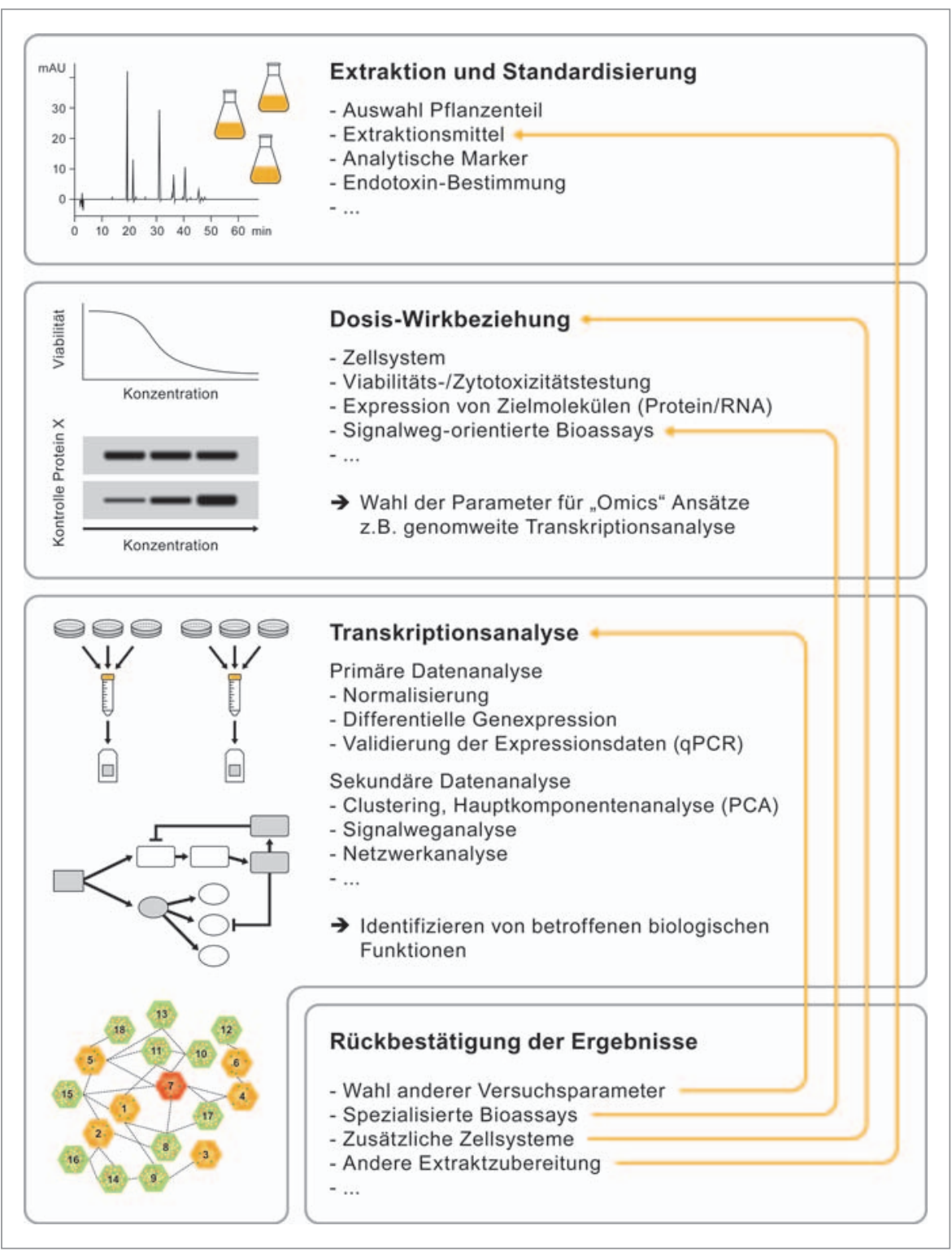

Abb. 1. Schematische Darstellung des experimentellen Designs einer In-vitro-Studie zur Wirkmusteranalyse von pflanzlichen Mehrstoffgemischen an Zellmodellen mittels Transkriptionsanalyse.
Weitere Studien zu Echinacea wurden in Tiermodellen, aber auch in weiteren In-vitro-Modellen durchgeführt. Beispielsweise untersuchten Chiu et al. [7] in einer Studie mit Lipopolysaccharid (LPS)-stimulierten THP-1-Monozyten, einem häufig verwendeten Entzündungsmodell, den Einfluss von Echinacea-Extrakt auf die Expression eines definierten Sets immunrelevanter Gene unter Verwendung von «fokussierten» Arrays. Derartige wissensbasierte Ansätze profitieren von den Ergebnissen aus gesamtgenomischen Ansätzen, da entsprechende Daten häufig frei zugänglich sind.

Weitere Studien zum Aktivitätsmonitoring von Mehrstoffgemischen mittels Transkriptionsanalyse sind z.B. die Analyse von Boswellia-Extrakt mithilfe von mikrovaskulären Endothelzellen [23], Artemisia iwayomogi in LPS-stimulierten gingivalen Fibroblasten [24] oder Heidelbeer-Extrakt (Vaccinium myrtillus) in der Makrophagen-ähnlichen Mauszelllinie RAW264 [25].

Vermehrt werden nicht nur Listen differenziell exprimierter Gene verwendet, um Rückschlüsse auf beteiligte Mechanismen zu ziehen, sondern auch speziellere bioinformatische Analysen wie etwa Clusterverfahren, Hauptkomponentenanalyse (PCA), Signal- 
weg- und Netzwerkanalysen [12]. Während Genlisten der Mehrdimensionalität der Signalweiterleitung nicht gerecht werden, wird bei der Signalweganalyse ein Aufspalten der Signalfolge in zwei oder mehrere Äste berücksichtigt [26]. In der Netzwerkanalyse werden Informationen von zusätzlichen Interaktionsdatenbanken mit dem eigenen Datensatz multidimensional in Verbindung gebracht.

\section{Diskussion}

In der Diskussion zu den Wirkmechanismen von Pflanzenextrakten oder -zubereitungen ist es notwendig, die multiplen Wirkmechanismen systematisch zu untersuchen. Die Vielzahl an zur Verfügung stehenden fokussierten Bioassays und die unzähligen Parameter, die in die Versuchsplanung mit einfließen, machen es jedoch nur schwer möglich, die Vorgehensweise in der Wirkmusterbeschreibung von Vielstoffgemischen exakt zu definieren.

Die vorgestellten Studien zu Ginkgo biloba-Extrakten, der Tibetischen Rezeptur Padma 28 und Echinacea-Extrakten zeigen das Potenzial der Transkriptionsanalyse zur Hypothesenbildung im Aktivitätsmonitoring. Eine Übersicht zur experimentellen Vorgehensweise bietet Abbildung 1. Rong et al. [27] diskutieren zudem die Verwendung der Transkriptionsanalyse an Zellsyste- men zur Erstellung eines «biologischen Response-Fingerabdrucks», d.h. zur Definition von Markern zur Kontrolle der biologischen Aktivität einer Rezeptur.

Eine direkte Extrapolation der In-vitro-Ergebnisse aus der Transkriptionsanalyse auf die In-vivo-Situation ist nicht möglich. Es können sich jedoch Hinweise auf unbekannte Mechanismen ergeben und somit weitere Untersuchungen gerechtfertigt werden, wie die Verwendung weiterer Zellmodelle oder anderer Konzentrationsbereiche. Dennoch sind jeder In-vitro-Analyse Grenzen gesetzt. Oft ist der zusätzliche Einsatz von Tiermodellen notwendig, um Rückschlüsse auf physiologische Prozesse zu ziehen. Trotz der niedrigeren Komplexität von Zellkulturen sind diese dennoch ein anerkanntes Hilfsmittel biomedizinischer Forschung [14].

Somit kann die Verwendung der beschriebenen Forschungsansätze eine Informationsbasis zur Charakterisierung von Mehrstoffgemischen bieten, die nach entsprechender Validierung sowohl in die Wirkmusterbeschreibung als auch in die Qualitätskontrolle von pflanzlichen Extrakten einfließen kann.

\section{Disclosure Statement}

Es bestehen keine Interessenskonflikte.

\section{Literatur}

1 Schmidt BM, Ribnicky DM, Lipsky PE, Raskin I: Revisiting the ancient concept of botanical therapeutics. Nat Chem Biol 2007:3:360-366.

2 Efferth T, Koch E: Complex interactions between phytochemicals. The multi-target therapeutic concept of phytotherapy. Curr Drug Targets 2011;12:122-132.

3 Schwabl H, Vennos C, Saller R: Tibetische Rezepturen als pleiotrope Signaturen - Einsatz von Netzwerk-Arzneien bei Multimorbidität. Forsch Komplementmed 2013;20(suppl 2):35-40.

4 Wink M: Evolutionary advantage and molecular modes of action of multi-component mixtures used in phytomedicine. Curr Drug Metab 2008;9:996-1009.

$\checkmark 5$ Schwabl H, Geistlich S, McHugh E: Tibetische Arzneimittel in Europa: historische, praktische und regulatorische Aspekte. Forsch Komplementmed 2006;13(suppl 1):1-6.

6 Bremner P, Heinrich M: Natural products as targeted modulators of the nuclear factor-kappaB pathway. J Pharm Pharmacol 2002:54:453-472.

7 Chiu SC, Tsao SW, Hwang PI, Vanisree S, Chen YA, Yang NS: Differential functional genomic effects of anti-inflammatory phytocompounds on immune signaling. BMC Genomics 2010;11:513.

8 Jenny M, Klieber M, Zaknun D, Schroecksnadel S, Kurz K, Ledochowski M, Schennach H, Fuchs D In vitro testing for anti-inflammatory properties of compounds employing peripheral blood mononuclear cells freshly isolated from healthy donors. Inflamm Res 2011;60:127-135.

-9 Sarkar FH, Li Y, Wang Z, Kong D: Cellular signaling perturbation by natural products. Cell Signal 2009;21:1541-1547.

10 Saller R, Melzer J, Rostock M: Antiinflammatory herbal drugs and their therapeutic potential in tumor patients. Forsch Komplementmed 2011;18: 203-212.

11 Ulrich-Merzenich G, Zeitler H, Jobst D, Panek D, Vetter H, Wagner H: Application of the '-Omic-' technologies in phytomedicine. Phytomedicine 2007; 14:70-82.
12 Gostner JM, Wrulich OA, Jenny M, Fuchs D, Ueberall F: An update on the strategies in multicomponent activity monitoring within the phytopharmaceutical field. BMC Complement Altern Med 2012;12:18.

13 Hudson J, Altamirano M: The application of DNA micro-arrays (gene arrays) to the study of herbal medicines. J Ethnopharmacol 2006;108:2-15.

14 Wrulich OA, Überall F: DNS-Mikroarrays - Verbesserung der analytischen Plattformen zur Beschreibung molekularer Wirkmuster von Naturstoffpräparaten oder Science Fiction? Schweiz Z Ganzheitsmed 2009;21:348-355.

15 Diamond BJ, Bailey MR: Ginkgo biloba: indications, mechanisms, and safety. Psychiatr Clin North Am 2013;36:73-83.

16 Smith JV, Burdick AJ, Golik P, Khan I, Wallace D, Luo Y: Anti-apoptotic properties of Ginkgo biloba extract EGb 761 in differentiated PC12 cells. Cell Mol Biol (Noisy-le-grand) 2002;48:699-707.

17 Klein A, Wrulich OA, Jenny M, Gruber P, Becker K, Fuchs D, Gostner JM, Uberall F: Pathway-focused bioassays and transcriptome analysis contribute to a better activity monitoring of complex herbal remedies. BMC Genomics 2013;14:133.

18 Vennos C, Melzer J, Saller R: Clinical studies on the efficacy and safety of Padma 28, a complex herbal formulation from Tibetan Medicine: an overview. Forsch Komplementarmed 2013;20 (suppl 2):25-30.

19 Melzer J, Brignoli R, Diehm C, Reichling J, Do DD, Saller R: Treating intermittent claudication with Tibetan Medicine PADMA 28: does it work? Atherosclerosis 2006;189:39-46.

20 Ueberall F, Fuchs D, Vennos C: Anti-inflammatory potential of Padma 28 - review of experimental data on the antiatherogenic activity and discussion of the multi-component principle. Forsch Komplementmed 2006;13(suppl 1):7-12.
1 Barnes J, Anderson LA, Gibbons S, Phillipson JD: Echinacea species (Echinacea angustifolia (DC.) Hell., Echinacea pallida (Nutt.) Nutt., Echinacea purpurea (L.) Moench): a review of their chemistry, pharmacology and clinical properties. J Pharm Pharmacol 2005;57:929-954.

22 Wang CY, Staniforth V, Chiao MT, Hou CC, Wu $\mathrm{HM}$, Yeh KC, Chen $\mathrm{CH}$, Hwang PI, Wen TN, Shyur LF, Yang NS: Genomics and proteomics of immune modulatory effects of a butanol fraction of Echinacea purpurea in human dendritic cells. BMC Genomics 2008;9:479.

23 Roy S, Khanna S, Shah H, Rink C, Phillips C, Preuss H, Subbaraju GV, Trimurtulu G, Krishnaraju AV, Bagchi M, Bagchi D, Sen CK: Human genome screen to identify the genetic basis of the anti-inflammatory effects of Boswellia in microvascular endothelial cells. DNA Cell Biol 2005;24:244-255.

24 Choi YG, Yeo S, Kim SH, Lim S: Anti-inflammatory changes of gene expression by Artemisia iwayomogi in the LPS-stimulated human gingival fibroblast: microarray analysis. Arch Pharm Res 2012;35:549-563.

25 Chen J, Uto T, Tanigawa S, Kumamoto T, Fujii M, Hou DX: Expression profiling of genes targeted by bilberry (Vaccinium myrtillus) in macrophages through DNA microarray. Nutr Cancer 2008;60 (suppl 1):43-50.

26 Werner T: Bioinformatics applications for pathway analysis of microarray data. Curr Opin Biotechnol 2008;19:50-54.

27 Rong J, Tilton R, Shen J, Ng KM, Liu C, Tam PK, Lau AS, Cheng YC: Genome-wide biological response fingerprinting $(\mathrm{BioReF})$ of the Chinese botanical formulation ISF-1 enables the selection of multiple marker genes as a potential metric for quality control. J Ethnopharmacol 2007;113:35-44. 